\title{
Transizioni dopo la scuola dell'obbligo: le scelte dei giovani in Ticino
}

\section{Elena Boldrini e Luca Bausch}

Nel contesto ticinese la propensione per la scelta di un percorso formativo con tirocinio in azienda è inferiore rispetto alla media nazionale, dove l'apprendistato duale risulta essere la via maggiormente praticata dai giovani in uscita dalle scuole dell'obbligo. Il presente contributo intende riferire di una ricerca pilota condotta nel Cantone Ticino in merito al passaggio dei giovani dal secondario I al secondario II, mirata alla comprensione delle modalità con cui questa transizione avviene e delle possibili problematicità insite in essa, relative soprattutto alla scelta di un percorso di formazione professionale con tirocinio in azienda.

Sono state rilevate le scelte, le ragioni, i criteri e le difficoltà (per mezzo del Career Decision Difficulties Questionnaire) nel processo di scelta al termine della quarta media di circa 170 allievi del Cantone, allargando il rilevamento dei dati anche ai loro stessi genitori, agli orientatori scolastico-professionali, ai coordinatori di sede delle scuole coinvolte e ai docenti di classe. Dai due rilevamenti di dati condotti (a distanza di sei mesi) emergono alcune piste di intervento per quanto concerne l'educazione alla scelta dei giovani, sia nelle sue specificità di sistema (organizzativoistituzionali), sia nelle sue declinazioni contenutistico-didattiche.

La transizione dal secondario I al secondario II in Svizzera: il contesto di riferimento.

La struttura del sistema formativo svizzero prevede, al termine del ciclo scolastico del secondario I (ISCED 21) sbocchi diversificati verso il sistema del secondario II (ISCED 3). Le diverse opportunità sono rappresentate a) dalle scuole che portano alla maturità liceale (della durata di 3 o 4 anni), b) dal settore professionale che comprende le scuole professionali a tempo pieno e la formazione duale con tirocinio in azienda. In entrambi i percorsi a carattere professionale si ottengono l'Attestato Federale di Capacità (AFC) ed è possibile accedere alla maturità professionale. L'ambito professionale offre pure la possibilità di una formazione pratica biennale che si conclude con un Certificato Federale di Formazione Pra- 
tica (CFFP). Completano queste piste principali le soluzioni dette "transitorie», rappresentate, ad esempio, da un decimo anno di formazione ed orientamento in attesa dell'inserimento in uno dei percorsi qui elencati ${ }^{2}$.

La transizione dal secondario I al secondario II (detta «transizione I») rappresenta un passaggio determinante per il futuro dei giovani e come tale esso è sempre più frequentemente oggetto di studi ed iniziative istituzionali (Pagnossin \& Armi, 2008) volte da un lato alla comprensione dei meccanismi che lo caratterizzano e delle implicazioni che esso assume a livello della struttura dell'intero sistema formativo; e, dall'altro, alla facilitazione di tale processo. In questa direzione la Conferenza Svizzera dei Direttori dell'Educazione pubblica (CDPE) ha lanciato nel 2006 il programma «Nahtstelle-Transition» 3 per l'ottimizzazione di questo passaggio, in collaborazione con la Confederazione e le Organizzazioni del Mondo del Lavoro. Lo studio TREE (Transition de l' Ecole à l'Emploi) ${ }^{4}$ ha lanciato un'inchiesta su un campione di circa 6000 giovani, rappresentativo a livello nazionale, volta a comprendere e descrivere i percorsi formativo-professionali dei giovani (TREE, 20035' Amos, 2007). Altri studi hanno messo in luce differenti aspetti di questo processo transitorio per quanto attiene le dinamiche individuali e psicosociali che lo caratterizzano (Herzog, Neuenschwander \& Wannack, 2006; Masdonati, 2007; Neuenschwander et al., 2005) e le sue ricadute di medio lungo termine (Donati, 1999, Donati \& Lafranchi, 2008).

Se questi programmi ed indagini sono attinenti alle modalità con cui si configura la domanda di formazione attraverso le scelte degli individui, un altro filone di ricerca non trascurabile - per la sua rilevanza soprattutto nel contesto della formazione professionale - è quello relativo all'analisi dell'offerta, soprattutto di quella del settore professionale, e specificamente all'analisi dell'offerta di posti di tirocinio, considerata in tutte le sue problematiche più propriamente economiche (Mühlemann, Schweri, Winkelmann \& Wolter, 2007; Wolter \& Schweri, 2004).

Il presente studio si inserisce in questo complesso e composito contesto di ricerca, rivolgendo l'attenzione in modo particolare all'esplorazione della natura e delle modalità di transizione dei giovani al termine della scuola dell'obbligo in Ticino, sullo sfondo delle peculiarità che contraddistinguono questa regione rispetto alla realtà nazionale, relativamente alla tipologia delle scelte compiute dai giovani e alla struttura del sistema formativo ${ }^{6}$. Alfine di comprendere l'intento del progetto che presenteremo nei paragrafi a seguire, occorre che si chiarisca quali siano le tendenze principali nell'ambito della transizione I a livello nazionale e le ragioni per le quali la realtà ticinese vi si discosta, soprattutto per quanto concerne i percorsi di formazione professionale duale.

La tradizione che caratterizza la cultura formativa svizzera ha fatto sì che si consolidasse nel tempo la prevalenza di scelte verso le formazioni professionali: attualmente, facendo riferimento ai dati dell'OFS (OFS, 20097), per quanto concerne il livello di formazione della popolazione svizzera compresa tra i 25 e i 
64 anni, la formazione professionale caratterizza il 45\% in media della stessa. A livello svizzero, all'incirca due terzi dei giovani sceglie un percorso professionale, contro un terzo che entra nelle cosiddette scuole di cultura generale (maturità). In particolare, secondo i dati OFS, circa il $65 \%$ in media sceglie un percorso professionale. Le differenze regionali sono però alquanto significative in quanto attestano per la Svizzera tedesca, nel 2007, una proporzione del $79.0 \%$ per la formazione professionale contro il $21.0 \%$ per la formazione culturale generale; per la Svizzera italiana invece la relazione è rispettivamente del $59.4 \%$ e del $40.6 \%$ e per quella francese del $58.4 \%$ e del $41.6 \%$ (OFS, $2009^{8}$ ).

Occorre pure segnalare ulteriori peculiarità che attualmente caratterizzano questo quadro rapidamente schizzato: i) la prima legata all'aumento, nell'ultimo quindicennio, dei tassi di frequentazione delle scuole di maturità, e ii) la seconda legata alla parallela diminuzione dei tassi di transizione diretta verso il settore professionale.

i) A metà degli anni '90, la formazione professionale ha visto infatti diminuire i propri allievi, a favore di un aumento parallelo della popolazione della formazione di cultura generale. Questo lo si può dedurre, ad esempio, dai dati relativi al numero di esami superati nell'ambito del secondario II: raffrontando i valori del 1990 con quelli del 2006, si registra un aumento del $7.1 \%$ relativamente alle formazioni di cultura generale e una diminuzione del $7.2 \%$ per la formazione professionale di base (Ghisla, Bonoli \& Loi, 2008, p.16) ${ }^{9}$.

ii) Un altro interessante aspetto riguarda l'indicatore del "tasso di transizione immediata", che misura la percentuale di allievi che, al termine del nono anno, hanno intrapreso direttamente una formazione professionale di base, una formazione generale o una soluzione intermedia (non certificante) ${ }^{10}$. Considerando il periodo dal 1990 al 2005, il tasso di transizione immediato è diminuito per la formazione professionale di più di 10 punti percentuali con una flessione particolarmente accentuata nel periodo 1995/1998. Per contro, è leggermente aumentato il grado di accesso immediato alla formazione di tipo generale. Il tasso di formazione transitoria è aumentato di ca. 5 punti (dal $9 \%$ al 14\%) ${ }^{11}$ (OFS, $2007 \mathrm{a}$, p. 47). A questo proposito occorre pure citare il dato relativo all'età media delle persone che accedono a soluzioni transitorie (decimo anno) che si è abbassata dal 1990 al 2006 di circa un anno: da 18.5 nel 1992 a 17.5 nel 2006 (OFS, 2007 b). Parallelamente si è alzata l'étà media di coloro che accedono ad una formazione professionale. Al contempo, come prevedibile, questa si è abbassata relativamente a coloro che intraprendono percorsi di formazione generale. Sempre più, dunque, alla fine della scuola dell'obbligo si verifica un accesso diretto ed immediato verso le scuole di cultura generale ed un effetto di prolungamento dello stato di transizione per coloro che poi, con grande probabilità, entreranno nel settore professionale (93\% di coloro che avevano intrapreso un percorso transitorio) facendo così aumentare l'età media della popolazione della formazione professionale. 


\section{Le peculiarità del Cantone Ticino}

Come si è già accennato, in questo quadro generale, il Canton Ticino presenta a sua volta una serie di peculiarità che possiamo riassumere schematicamente con: i) una più marcata discesa nell'ultimo quindicennio - rispetto al dato nazionale - della frequentazione della formazione professionale; ii) un elevato tasso di accessi diretti alle scuole di maturità per coloro che posseggono i requisiti richiesti; iii) una composizione della popolazione che frequenta le scuole professionali con tassi di bocciature - all'interno dell'intera biografia scolastica - ed un'età media di gran lunga più elevati rispetto agli altri ordini di scuola; iv) una notevole disparità di transizioni verso il settore professionale nelle diverse regioni del Cantone; v) un elevato tasso di abbandoni nei percorsi liceali; vi) e una presenza minoritaria, rispetto agli altri Cantoni, di soluzioni «transitorie».

i) Se nella Svizzera tedesca, come si è visto, il tasso di giovani che nel 2007 hanno scelto un percorso di tipo professionale era del 79\%, questo dato, per il Ticino, scende al 59.4\%. Se si considera poi l'ultimo quindicennio, si nota un notevole abbassamento del tasso di scelta per il tirocinio in azienda (scuole professionali secondarie a tempo parziale) dal 35.9\% del 1991/92, al 26.3\% del 2006/07, pari ad una diminuzione di circa il 10\%. In particolare per le professioni artigianali e industriali (-4.2) e, ancora di più, per quelle di tipo commerciale $(-7.1)^{12}$. Al contempo le SMS (Scuole Medie Superiori) hanno visto un aumento del $6.2 \%$, e le scuole professionali a tempo pieno un aumento del 6\% (Guidotti \& Rigoni, 2007a, p. 7).

ii) Vi è una marcata propensione degli svizzeri, delle ragazze, di coloro che non hanno mai subito bocciature, a frequentare le scuole medie superiori (SMS). Secondo i dati di "Scuola a tutto campo»(USR, 2007, p. 89), in Ticino, quasi il $50 \%$ degli allievi ha la possibilità di frequentare una SMS senza esami di ammissione $^{13}$ : la quasi totalità di questi frequenta una SMS (licei e scuola cantonale di commercio); soltanto l'8\% di questi sceglie invece altri percorsi.

Ciò risulta facilitato, inoltre, dalla particolare strutturazione del sistema formativo ticinese, tale per cui la scuola media ha un carattere molto meno selettivo rispetto alle scuole dello stesso ordine e grado del resto della Svizzera.

iii) Secondo i dati del censimento degli allievi per l'anno 2006/07 (Guidotti \& Rigoni, 2007b), analogamente al dato nazionale, alla fine della quarta media la condizione socio-economica, il sesso, la nazionalità, il numero di classi ripetute, giocano un ruolo importante nella definizione dei percorsi della formazione post-obbligatoria. La formazione professionale presenta il maggior tasso di popolazione che ha ripetuto una o più classi nel proprio percorso scolastico $(6.5 \%$ nel 2006/2007 secondo i dati del «Censimento degli allievi 2006/2007», p. 8). Inoltre il censimento degli allievi 2006/2007, rileva come la formazione profes- 
sionale a tempo parziale sia il settore con l'età più alta per il medesimo livello formativo: il 36.4\% degli allievi ha 19 anni o più ${ }^{14}$, contro il $9.9 . \%$ delle scuole medie superiori presentano e, rispettivamente, il 20.9\% delle scuole professionali a tempo pieno.

iv) Il fattore territoriale risulta essere particolarmente determinante: per l'anno scolastico 06/07 i territori delle valli presentano una marcata propensione per la scelta del tirocinio in azienda (40.2\%) ed una minore per le SMS (28.7\%), mentre quelli urbani si comportano in modo opposto con oltre il $40 \%$ di scelte per le SMS (UOSP, 2008). La percentuale media a livello cantonale di preferenze per le SMS e la scuola professionale a tempo pieno rappresenta il $60.78 \%$, dato che rende evidente l'adesione assai minoritaria per la formazione professionale duale $(28.56 \%)$.

v) I dati USTAT ${ }^{15}$ relativi agli abbandoni e alle bocciature nei licei cantonali, suggeriscono un ulteriore elemento da tenere in considerazione in questa panoramica: dei 1070 allievi del primo anno (anno 2003/04) il 30\% ca. non conclude il percorso di formazione (anno 2006/2007).

vi) Inoltre secondo lo studio di Egger, Dreher \& Partners (2007), la diversificazione delle soluzioni transitorie in Ticino risulta anch'essa peculiare: se nei cantoni della Svizzera tedesca la presenza di soluzioni transitorie di tipo scolastico e la diversificazione delle misure transitorie in generale risulta essere particolarmente marcata, la situazione ticinese mostra una minore diversificazione e una minore possibilità di continuare su di un percorso scolastico preparatorio ad una reale maturazione della scelta professionale.

Questo quadro, al contempo complesso e peculiare, ha aperto la strada a diverse interpretazioni ipotetiche del «Sonderfall» ticinese (Ghisla, 2003). Ne riassumiamo le principali, nella misura in cui forniscono la tela di fondo per la costruzione e l'interpretazione della ricerca qui in oggetto. Esisterebbe una propensione culturale ${ }^{16}$ verso le Scuole Medie Superiori (SMS) e più in generale per la formazione scolastica in senso stretto. Parallelamente la scelta del tirocinio in azienda viene percepita come una soluzione di ripiego perché non direttamente propedeutica alle professioni di "alto profilo» (considerate la via per la realizzazione e per il successo); osservazione che a sua volta implicherebbe una scarsa conoscenza del mondo dei mestieri e più in generale del lavoro. Inoltre la scelta di scuole di cultura generale permetterebbe di procrastinare la scelta di un mestiere o di una professione, o perlomeno la chiara formazione di una rappresentazione professionale del giovane. 


\section{Il progetto di ricerca}

\section{Il quadro teorico-concettuale di riferimento}

Cercare di tratteggiare un quadro dei processi che caratterizzano la transizione dalla quarta media al mondo formativo o professionale successivo, significa interessarsi al fenomeno di transizione in quanto passaggio. L'analisi conoscitiva sul terreno è stata messa in atto facendo riferimento a prospettive psico-sociali relativamente al concetto di transizione post-secondario I (Herzog et al., 2006) ${ }^{17}$ che tengono conto sia di una serie di elementi esogeni e contestuali - relativi alle variabili di tipo socio-demografico, alle interazioni che il soggetto ha nel suo contesto di riferimento (famiglia, scuola, orientamento, gruppo dei pari) -, sia di elementi di tipo endogeno e personale, legati primariamente alla psicologia, alle credenze e agli obiettivi propri del singolo soggetto. Il processo di transizione è infatti insieme un processo intra-individuale e inter-individuale (Albert, 2007; Masdonati, 2007) che può essere compreso solamente considerando i diversi elementi che lo compongono: il contesto, i meccanismi di appropriazione individuale della transizione (rappresentazioni di sé, delle competenze richieste, delle professioni ideali).

La rassegna tematica sulle ricerche svizzere relative al tema della transizione tra la formazione e il mondo del lavoro (Pagnossin \& Armi, 2008) dopo il 1980, ha messo in evidenza come le diverse ricerche condotte - se considerate dal punto di vista degli attori coinvolti nella transizione - siano riconducibili alla centralità di tre soggetti: a) i giovani; b) i rappresentanti del sistema educativo; c) i rappresentanti del mondo del lavoro. In base agli elementi sopracitati e agli assi di ricerca esplorati, è parso importante considerare, nel nostro studio, sia le variabili individuali (endogene), sia quelle più propriamente sociali (esogene), andando a rilevare non solo il punto di vista dei giovani, ma anche quello degli altri attori che ruotano intorno al loro processo di transizione: i loro stessi genitori (prospettiva quest'ultima che pare non avere grande risalto nella letteratura esistente), i docenti, gli orientatori professionali e i coordinatori di sede dell'orientamento ${ }^{18}$.

\section{Obiettivi della ricerca}

A partire da queste considerazioni, il progetto di ricerca-azione SCELTOplus ${ }^{19}$, si è posto l'obiettivo di indagare le modalità delle scelte verso il secondario II in Ticino, enucleando una serie di possibili «ambiti problematici», passibili di interventi mirati (informativo, formativo, di orientamento, organizzativo...), con il fine ultimo di ipotizzare delle strategie sperimentali di intervento mirate al riequilibrio della domanda di tirocinio in Ticino.

Vi sono, in particolare, tre ordini di obiettivi del progetto: un obiettivo più ampio e generale che è quello di rendere maggiormente attrattivo l'apprendistato alfine di aumentare il numero e la motivazione dei giovani che vi accede; un ob- 
iettivo specifico che è quello conseguente di elaborare e sperimentare una strategia di promozione del tirocinio in azienda (misure sperimentali di intervento); un obiettivo specifico di ricerca - oggetto, quest'ultima, di una sintesi in questa sede - che è quello di comprendere come avviene il processo di transizione e di scelta tra il secondario inferiore e il secondario superiore.

\section{Design di ricerca}

\section{GL i ambiti indagati}

Da quanto esposto precedentemente, occorre tenere in considerazione le variabili socio-culturali all'interno delle quali ha luogo la transizione e di cui il soggetto, in un certo senso, "subisce» il condizionamento, così come occorre ricordare al contempo che vi sono delle dinamiche psicologiche ed individuali che il soggetto «attiva». L'ordine delle riflessioni psico-sociali risulta dunque una chiave interpretativa ricca che considera oltre alla complessità degli elementi individuali (endogeni), anche le condizioni di contesto entro cui esse si giocano (esogeni). Il modello proposto da Masdonati (2007, p. 21) riassume queste riflessioni tenendo conto di tre ordini di elementi: a) il contesto in cui avviene la transizione, descrivibile per mezzo delle condizioni socio-economiche e socio-biografiche; b) l' «appropriazione individuale della transizione», ovvero delle variabili individuali (percezione di sé, della transizione, delle proprie attitudini e delle competenze richieste in specifici contesti formativo/professionali); c) i risultati della transizione a breve (inserimento) medio (transizione) lungo (mantenimento) termine, secondo criteri di soddisfazione per rapporto all'inserimento e al mantenimento.

Sulla base di questo approccio teorico-concettuale, il design dell'indagine sul terreno era teso al rilevamento di dati relativi ai seguenti ambiti:

i) condizioni socio-demografici dei giovani;

ii) rappresentazioni personali della professione ideale ${ }^{20}$;

iii) stato della scelta al termine della quarta media e livello di indecisione professionale (Gati, Krausz, Osipow, 1996);

iv) scelta effettuata (o ipotesi di scelta) per il secondario II e ragioni soggiacenti;

v) ostacoli che potrebbero compromettere la scelta effettuata;

vi) ipotetica «seconda scelta»;

vii) conoscenza delle fonti di informazione e loro utilità percepita;

viii) ricadute e stato della scelta ad un semestre di distanza dalla scelta stessa.

\section{Il campione}

Il campione delle tre sedi di scuola media coinvolte è stato selezionato sulla base di precise peculiarità proprie delle eterogeneità territoriali del Cantone Ticino, 
così da poter indagare eventuali diversità a livello delle modalità di scelta dei giovani $^{21}$.

All'interno di queste sedi scolastiche sono stati considerati come soggetti dell'indagine:

- gli allievi di 3 classi per ogni sede considerata;

- i loro genitori;

- i docenti di classe;

- i coordinatori di sede (antenne dell'orientamento);

- gli orientatori scolastici e professionali (dell'Ufficio Orientamento Scolastico e Professionale - UOSP).

\section{Raccolta de $i$ dati}

L'indagine si è realizzata in due fasi: una fase ex ante (al termine della quarta media dell'anno scolastico 2007/08; Cfr. Tabella 1) ed una fase ex post (all'inizio dell'anno scolastico 2008/2009; Cfr. Tabella 2).

- Un questionario principale concernente gli aspetti sopra citati - fatta eccezione per il punto viii) che è stato indagato nel corso della somministrazione di un secondo questionario al medesimo gruppo di giovani - è stato concepito e somministrato nel giugno 2008 ad indirizzo di un gruppo di allievi di quarta media afferenti alle tre sedi di scuola media menzionate.

- Tale questionario é stato «replicato» in quelle parti che potevano apportare informazioni importanti (conferme, differenze di rappresentazioni, ...) per i relativi genitori e docenti di classe dei giovani interpellati.

- Inoltre gli orientatori scolastici e professionali e i coordinatori di sede (con incarichi di orientamento) delle sedi sono stati intervistati sulle medesime tematiche oltre che sulle loro pratiche professionali, facendo capo ad interviste semi-strutturate.

Tabella 1. Campione, metodo di indagine e tipologia di analisi della fase ex-ante (giugno 2008).

\begin{tabular}{|c|c|c|c|c|c|c|}
\hline \multicolumn{7}{|c|}{ Indagine ex ante } \\
\hline \multirow{5}{*}{ 䒕 } & sede scolastica & allievi & genitori & docenti & $\begin{array}{l}\text { Coordinatori } \\
\text { di sede }\end{array}$ & orientatori \\
\hline & scuola medial & 50 & 48 & 3 & 1 & 1 \\
\hline & scuola media2 & 65 & 58 & 1 & 1 & 1 \\
\hline & scuola media3 & 57 & 50 & 3 & 1 & 1 \\
\hline & $T O T$ & 172 & $156^{22}$ & 7 & 3 & 3 \\
\hline $\begin{array}{l}\text { Metodo } \\
\text { indagine }\end{array}$ & & \multicolumn{3}{|c|}{$\begin{array}{l}\text { Somministrazione di un } \\
\text { questionario «simmetrico» }\end{array}$} & \multicolumn{2}{|c|}{ Interviste semi-strutturate } \\
\hline Analisi & & \multicolumn{3}{|c|}{ Quantitativa } & \multicolumn{2}{|l|}{ Qualitativa } \\
\hline
\end{tabular}


La fase ex-post (Cfr. Tabella 2) ha previsto un secondo questionario, a distanza di un semestre scolastico, somministrato ai medesimi giovani del campione della fase ex-ante, al fine di verificare i) se la scelta effettuata al termine dell'anno scolastico precedente è stata poi effettivamente concretizzata, ii) se la scelta effettuata sembra soddisfare il giovane e iii) se le rappresentazioni professionali dei giovani sono nel frattempo cambiate. Il 68.9\% (126 allievi su 183) degli stessi allievi ha risposto anche al secondo questionario; il $25 \%$ ha partecipato solo alla prima fase e il $4.9 \%$ solo alla seconda fase.

Tabella 2. Campione, metodo di indagine e tipologia di analisi della fase ex-post (novembre 2008).

\begin{tabular}{|l|l|c|}
\hline \multicolumn{3}{|c|}{ Indagine ex post } \\
\hline \multirow{3}{*}{} & Ex allievi scuola media & $\begin{array}{c}\text { N rispondenti } \\
\text { entrambe le fasi; } \%\end{array}$ \\
\cline { 2 - 3 } & scuola medial & $38(73.1 \%)$ \\
\cline { 2 - 3 } & scuola media 2 & $46(68.7 \%)$ \\
\cline { 2 - 3 } & scuola media3 & $42(65.6 \%)$ \\
\cline { 2 - 3 } & TOT & $126(69 \%)$ \\
\hline \multirow{2}{*}{ Metodo di indagine } & \multicolumn{2}{|c|}{ Somministrazione di un questionario } \\
\hline Analisi & \multicolumn{2}{|c|}{ Quantitativa } \\
\hline
\end{tabular}

\section{Risultati in sintesi dell'analisi quantitativa}

Veniamo, qui di seguito, alla presentazione dei principali risultati rispetto a quanto emerso sia dalla fase ex-ante, sia da quella ex-post, in merito ad alcuni degli ambiti di cui sopra.

\section{Le rappresentazioni personali della professione ideale}

Per entrare nel merito delle rappresentazioni professionali dei giovani ${ }^{23}$, è stato loro chiesto di indicare la professione che ritengono più adatta per il loro futuro lavorativo. La stessa domanda è stata posta ai loro genitori, in relazione al futuro professionale dei figli. Ne emerge una tendenza marcata alla scelta di professioni intellettuali e scientifiche, sia da parte dei genitori, sia, in maniera più evidente, da parte dei giovani. Se soltanto il 10.7\% (Cfr. Tabella 3) dei genitori esercita una professione intellettuale o scientifica ${ }^{24}$ ben il $23.5 \%$ dell'insieme dei genitori stessi pensa che una professione intellettuale o scientifica sia ideale per il figlio (Cfr. Tabella 425 ) e ben il 30.2\% dei figli stessi la considera la più adatta a loro $^{26}$ (Cfr. Tabella 527). Per questa categoria professionale, si osserva una «amplificazione» dei progetti professionali, sia da parte dei genitori, sia, soprattutto da parte dei figli: per i mestieri di tipo amministrativo, per le professioni inter- 
medie e per il personale dei servizi/vendita, la tendenza è opposta; meno della metà dei genitori reputa la loro stessa professione adatta al figlio e ancor meno $\mathrm{i}$ loro figli. La sede scolastica situata nel distretto urbano arriva ad una percentuale del $34 \%$ di giovani che vorrebbe esercitare un professione intellettuale o scientifica e quella della zona di confine al 37\% (è la sede «urbana» che registra la percentuale più alta $-17.5 \%$ - di genitori con professioni intellettuali/scientifiche). Al contrario è la scuola media di valle che registra il $20 \%$ di giovani che desiderano intraprendere mestieri di tipo artigianale/industriale (la sede di valle ha una percentuale del $2 \%$ di genitori che esercitano professioni intellettuali e scientifiche $\left.{ }^{28}\right)$.

Tabella 3. Percentuali relative alle professioni esercitate dai genitori rispondenti.

\begin{tabular}{|c|c|c|c|c|c|}
\hline $\begin{array}{l}\text { Professione del genitore che } \\
\text { compila il questionario }\end{array}$ & Sme1 & Sme2 & Sme3 & Totale & p-valore \\
\hline $\begin{array}{l}\text { Professioni intellettuali e } \\
\text { scientifiche }\end{array}$ & $2.1 \%$ & $17.5 \%$ & $11.6 \%$ & $10.8 \%$ & \multirow{10}{*}{$P=0.018^{29}$} \\
\hline Professioni intermedie & $10.4 \%$ & $24.6 \%$ & $27.9 \%$ & $20.9 \%$ & \\
\hline Impiegati di tipo amministrativo & $16.7 \%$ & $12.3 \%$ & $16.3 \%$ & $14.9 \%$ & \\
\hline Personale dei servizi e venditori & $27.1 \%$ & $22.8 \%$ & $4.7 \%$ & $18.9 \%$ & \\
\hline \begin{tabular}{|l}
$\begin{array}{l}\text { Agricoltori e operai qualificati } \\
\text { (agricoltura e della pesca) }\end{array}$ \\
\end{tabular} & $.0 \%$ & $1.8 \%$ & $.0 \%$ & $.7 \%$ & \\
\hline $\begin{array}{l}\text { Artigiani e operai (mestieri di } \\
\text { tipo artigianale }\end{array}$ & $12.5 \%$ & $5.3 \%$ & $.0 \%$ & $6.1 \%$ & \\
\hline $\begin{array}{l}\text { Operai specializzati operai } \\
\text { dell'assemblaggio }\end{array}$ & $2.1 \%$ & $1.8 \%$ & $2.3 \%$ & $2.0 \%$ & \\
\hline Operai non qualificati & $2.1 \%$ & $1.8 \%$ & $7.0 \%$ & $3.4 \%$ & \\
\hline Casalinga & $25.0 \%$ & $10.5 \%$ & $30.2 \%$ & $20.9 \%$ & \\
\hline Altro & $2.1 \%$ & $1.8 \%$ & $.0 \%$ & $1.4 \%$ & \\
\hline
\end{tabular}

In relazione alla professione o al mestiere ideale è stato chiesto a genitori e figli di indicare quale sia la funzione principale assolta dal mestiere nella vita (Cfr. Grafico 1) («La professione che un giorno farò dovrà permettermi di...»). Sia per i giovani, sia per i loro genitori, sia per i docenti di classe la funzione considerata predominante è «soddisfare tutte le aspirazioni» $(5.95,5.05,6.7$ di punteggio medio rispettivamente per genitori, figli e docenti, su una scala da 1 a7). Se per i giovani segue poi l'»avere una sicurezza economica dignitosa» (Media $=4.49)$ e "avere un posto di lavoro fisso" (Media=4.38), per i loro genitori e per i docenti appare più importante «esprimere le attitudini personali» (Media genitori=5.13; Media docenti=6.0). Appare così predominante nei giovani, ma sopratutto nei genitori, l'aspettativa per una realizzazione personale totale nella professione: una realizzazione delle proprie aspirazioni, un'espressione delle attitudini personali. Dato, quest'ultimo, che supporterebbe quanto visto a proposito dell'alto tasso di giovani che desiderano intraprendere professioni accademiche. 
Tabella 4. Professioni che i genitori ritengono ideali per i loro figli

\begin{tabular}{|l|c|}
\hline $\begin{array}{l}\text { Professione che il genitoreritiene } \\
\text { ideale per il figlio }\end{array}$ & Totale \\
\hline Forze armate & $0 \%$ \\
\hline $\begin{array}{l}\text { Professioni intellettuali e } \\
\text { scientifiche }\end{array}$ & $23.5 \%$ \\
\hline Professioni intermedie & $14.4 \%$ \\
\hline Impiegati di tipo amministrativo & $6.8 \%$ \\
\hline Personale dei servizi e venditori & $5.3 \%$ \\
\hline $\begin{array}{l}\text { Agricoltori e operai qualificati } \\
\text { agricoltura e della pesca) }\end{array}$ & $0 \%$ \\
\hline $\begin{array}{l}\text { Artigiani e operai (mestieri di } \\
\text { tipo artigianale }\end{array}$ & $13.6 \%$ \\
\hline $\begin{array}{l}\text { Operai specializzati operai } \\
\text { dell'assemblaggio }\end{array}$ & $0.8 \%$ \\
\hline Operai non qualificati & $0 \%$ \\
\hline Casalinga & $0 \%$ \\
\hline Altro & $0 \%$ \\
\hline
\end{tabular}

Tabella 5. Professioni che i giovani considerano piu adatte a loro.

\begin{tabular}{|l|c|}
\hline $\begin{array}{l}\text { Professione che il giovane } \\
\text { considera più adatta a sé }\end{array}$ & Totale \\
\hline Forze armate & $0.6 \%$ \\
\hline $\begin{array}{l}\text { Professioni intellettuali e } \\
\text { scientifiche }\end{array}$ & $30.2 \%$ \\
\hline Professioni intermedie & $23.3 \%$ \\
\hline Impiegati di tipo amministrativo & $5.2 \%$ \\
\hline Personale dei servizi e venditori & $7.0 \%$ \\
\hline $\begin{array}{l}\text { Agricoltori e operai qualificati } \\
\text { (agricoltura e della pesca) }\end{array}$ & $1.2 \%$ \\
\hline $\begin{array}{l}\text { Artigiani e operai (mestieri di } \\
\text { tipo artigianale }\end{array}$ & $15.7 \%$ \\
\hline $\begin{array}{l}\text { Operai specializzati operai } \\
\text { dell'assemblaggio }\end{array}$ & $0 \%$ \\
\hline Operai non qualificati & $0 \%$ \\
\hline Casalinga & $0 \%$ \\
\hline Altro & $0 \%$ \\
\hline
\end{tabular}

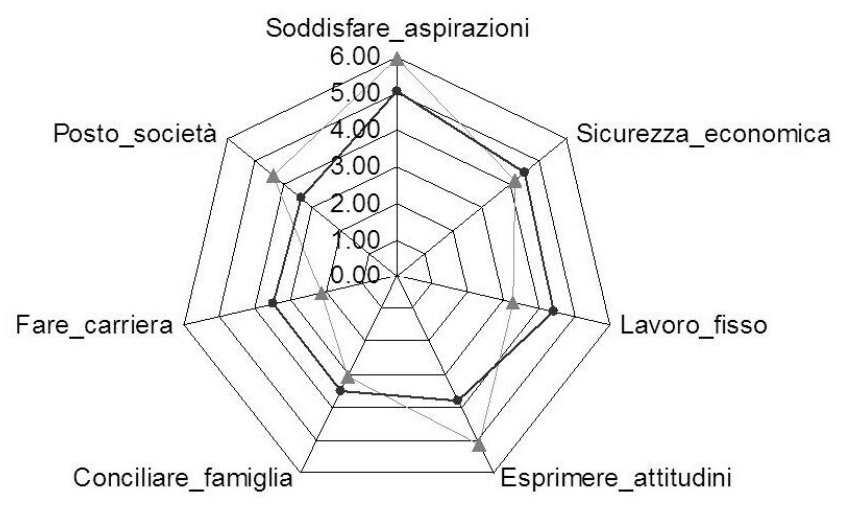

Grafico 1. A quali funzioni assolve la professione? Punteggi medi per genitori e figli classificati su una scala da 1 a 7.

\section{Quali difficoltà nella scelta?}

Si è poi misurato lo stato della decisione formativa o professionale per il «dopo scuola media» e le diverse difficoltà che possono intercorrere nell'ambito della scelta. Per quanto concerne lo stato della scelta, il 10\% ${ }^{30}$ dei giovani - al mese di giugno dell'anno scolastico 2007/8 - «non ha alcuna idea di cosa farà» dopo la scuola media, mentre il $60 \%$ ha fatto una scelta definitiva e la restante parte deve ancora confermare l'ipotesi o scegliere tra una rosa di possibili soluzioni. 
Per quanto attiene la misurazione delle difficoltà nella presa di decisione relativa alla carriera, esse sono state rilevate per mezzo dell'utilizzo della tassonomia di Gati, Krausz e Osipow, (1996) e del relativo questionario "Career Decision-Making Difficulties Questionnaire» (CDDQ). L'indecisione professionale (Vocational Indecision) è un concetto che fa riferimento alle difficoltà che eventualmente un soggetto può incontrare nel momento in cui è chiamato ad operare una scelta che concerne la sua carriera Il CDDQ si basa su una tassonomia di queste difficoltà, su tre livelli di categorizzazione: la prima distinzione intercorre tra le difficoltà che possono presentarsi prima dell'effettivo inizio del processo di presa di decisione, e quelle difficoltà che si presentano durante il processo di presa di decisione. In quest'ultima categoria si distingue poi tra le difficoltà relative nell'utilizzare informazioni disponibili per la presa di decisione e quelle relative all'effettiva mancanza di informazioni. Questa prima tripartizione è poi a sua volta dettagliata da dieci sotto-componenti, ciascuna delle quali viene misurata nel questionario del CDDQ per mezzo di una serie di items, con scala Likert a 9 punti.

Tali items fanno riferimento alle seguenti componenti e sottocomponenti: 1) Lack of Readiness ${ }^{31}(\mathrm{R})$ - che a sua volta include: la mancanza di motivazione $(\mathrm{Rm})$, l'indecisione cronica (Ri), e le credenze o miti disfunzionali (Rd); 2) Lack of Information $32(\mathrm{M})$ - che a sua volta include: mancanza di informazioni sul processo $(\mathrm{Mp})$, mancanza di informazioni sul sé $(\mathrm{Ms})$, mancanza di informazioni sui mestieri $(\mathrm{Mm})$, difficoltà ad ottenere le informazioni (Mo); 3) Inconsistent Information $^{33}$ (I) - che a sua volta include: informazioni incoerenti (unreliable) (Iu), conflitti interni (Ii), e conflitti esterni (Ie).La media dei valoroi ottenuti per le tre componenti principali (R, M, I) costituisce il punteggio della scala complessiva di indecisione professionale.

Il punteggio medio della scala complessiva di indecisione professionale (Vocational Indecision - VI) è abbastanza moderato ${ }^{34}($ Media=3.5) e non presenta differenze significative al suo interno per quanto concerne le diverse sedi, il sesso, la nazionalità, la lingua madre, le bocciature, e i corsi attitudinali frequentati. Più diversificato il panorama delle diverse scale e sottoscale che presentiamo qui di seguito.

Per quanto concerne la scala Lack of Readiness (R), il Grafico 2 ci mostra come quelli che vengono definiti miti disfunzionali o credenze irrazionali (Rd; Me$\mathrm{dia}=4.94)$ presentano un punteggio relativamente alto e quindi un sensibile impatto sulla difficoltà nella scelta. Con questa sottoscala si misura la propensione del soggetto a sviluppare credenze, idealizzazioni e miti irrealistici relativamente alla natura, alle conseguenze, all'irreversibilità delle sue scelte professionali. $\mathrm{Si}$ tratta di aspettative troppo elevate, come per esempio il fatto di credere che una professione possa realizzare tutte le aspirazioni di una persona (dato che conferma quanto mostrato nel Grafico 1) o risolvere tutti i suoi problemi. 


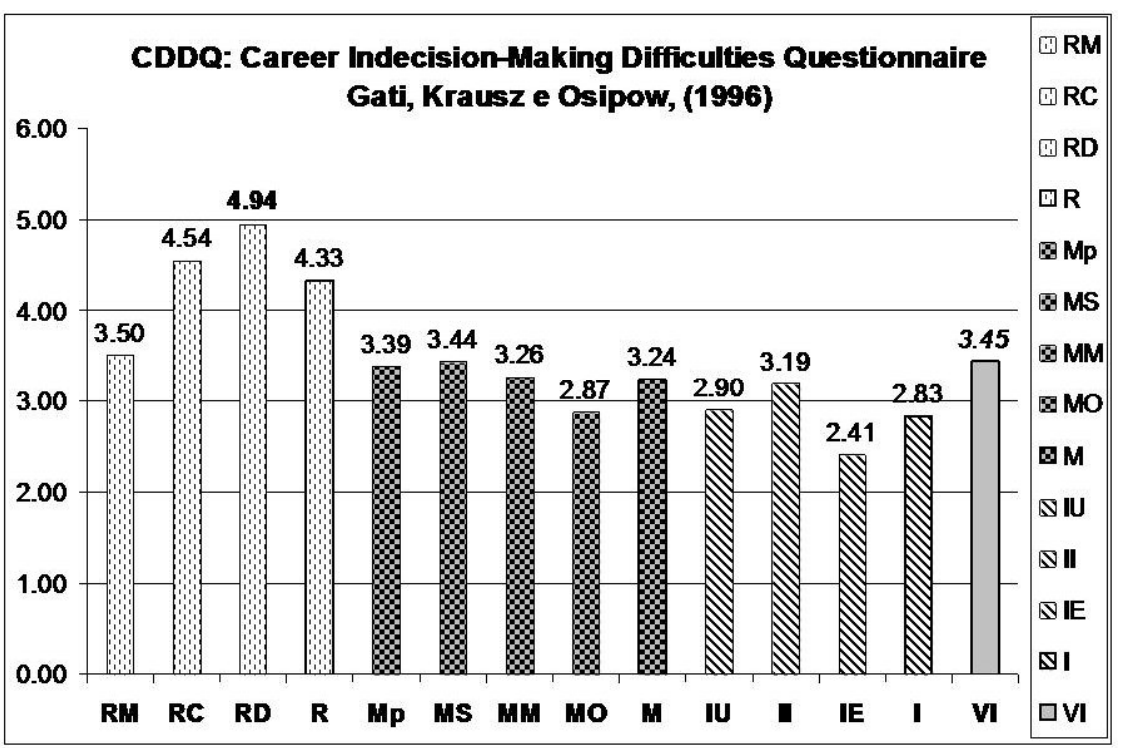

Grafico 2. Medie per le scale di $R$ (Readiness), $M$ (Lack of information) e I (Inconsistent information), e relative sottocomponenti.

Tali miti disfunzionali si presentano in maniera significativamente diversa in base al sesso $(\mathrm{p}=0.045)$, e alla nazionalità $(\mathrm{p}=0.022)$. Coloro che sono di nazionalità straniera presentano una media su questa sottoscala pari a 5.52 (gli svizzeri 4.80; $\mathrm{p}=0.022)$ e i maschi una media di 5.19, rispetto alle femmine: $4.69(\mathrm{p}=0.045)^{35}$. Inoltre, per quegli items del CDDQ che potevano essere replicati anche nel questionario dei genitori, abbiamo verificato quanto le risposte dei genitori si discostassero da quelle dei figli; il loro raffronto permette di comprendere che la visione di figli e genitori è omogenea e molto elevata per quanto concerne, proprio, un item relativo alle credenze disfunzionali: «mi aspetto che la formazione/professione che sceglierò, mi permetterà di realizzare tutte le mie aspirazioni»(Media genitori=7.8; Media figli=7.3).

La mancanza di motivazione $(\mathrm{Rm})$ a prendere una decisione è significativamente più elevata nei maschi (3.74) che nelle femmine (3.27; $\mathrm{p}=0.045)$. In generale coloro che sono di nazionalità straniera sono meno pronti a prendere una decisione relativamente alla loro carriera, rispetto ai loro compagni svizzeri $(\mathrm{p}=0.019)^{36}$.

Per quanto concerne la mancanza di informazioni (M), la media globale di 3.24 presenta al suo interno una differenza significativa relativamente a coloro che hanno già avuto una bocciatura nel corso del loro curriculum scolastico: essi denotano una mancanza di informazioni più alta (4.00) rispetto ai loro compagni 
(3.11; $\mathrm{p}=0.015)$. Questo gruppo si caratterizza, proporzionalmente al resto del campione, per una minore informazione sui diversi mestieri (4.19; $\mathrm{p}=0.011) \mathrm{e}$ per una più marcata difficoltà nel reperimento di informazioni sia su di sé, sia sui mestieri, sia sui percorsi formativi $(3.62$; $\mathrm{p}=0.024)$. Punteggi medi moderatamente alti si riscontrano anche nella mancanza di informazioni sul processo di scelta (come si fa la scelta professionale formativa, quali variabili essa comprende) (Media=3.39), nella conoscenza di se stessi e delle proprie attitudini e competenze (3.44), così come nella conoscenza dei mestieri (3.26).

La scala relativa alle possibili informazioni inconsistenti, ovvero ad una possibile contraddittorietà tra le diverse informazioni e fonti di informazione è mediamente bassa, ma in ogni caso significativamente maggiore per coloro che hanno già ripetuto un anno: essi presentano un problema di unreliable information maggiore rispetto ai loro compagni (3.65 e 2.77; $p=0.021$ ); essi hanno maggiori conflitti esterni (gli attori di riferimento del giovane hanno preferenze diverse da quelle del giovane stesso) $(3.32 ; \mathrm{p}=0.012)$ e in maniera particolare i maschi rispetto alle loro compagne femmine $(\mathrm{p}=0.049)$. In generale però la misura dei conflitti esterni è la più bassa del CDDQ: questo ha a che fare evidentemente con l'assenza di conflittualità e di dibattito dialettico tra genitori e figli in fase di scelta. Alla domanda "è d'accordo con la scelta o ipotesi di scelta di suo figlio?» il $100 \%$ dei genitori rispondenti, afferma di essere d'accordo.

\section{La scelta effettuata}

Le scelte formative degli allievi presentano per il $42.9 \%$ una preferenza per una SMS. Il 22\% vorrebbe intraprendere una formazione professionale con tirocinio in azienda, mentre il 10\% una scuola professionale a tempo pieno. Il 15\% presenta una indecisione tra due ipotesi. Come è evidente (Cfr. Tabella 6), le percentuali di queste scelte variano in maniera significativa in base alla sede scolastica: la sede di valle presenta la minor percentuale di ragazzi (30\%) che decidono di frequentare una SMS, e la più alta per il tirocinio in azienda con un 34.7\%. Nella sede del distretto urbano il 44.6\% decide per una SMS e nella sede di confine ben il 51.8. Il 6.5\% si propone di seguire stages all'estero o prendere altre direzioni e l'1.2\% ha già deciso di ripetere la quarta media. Se alla percentuale del totale delle preferenze per le SMS aggiungiamo anche coloro che presentano anche una seconda opzione e quindi sono indecisi tra una SMS e un'altra opzione, otteniamo l'alta percentuale del $54.8 \%$. 
Tabella 6. Percentuali delle scelte in totale, differenziate per sede scolastica.

\begin{tabular}{|l|c|c|c|c|}
\hline & Sede1 & Sede2 & Sede3 & Totale \\
\hline SMS & $30.6 \%$ & $44.6 \%$ & $51.8 \%$ & $42.9 \%$ \\
\hline SPTP & $6.1 \%$ & $10.8 \%$ & $12.5 \%$ & $10.0 \%$ \\
\hline SPTA & $34.7 \%$ & $16.9 \%$ & $17.9 \%$ & $22.4 \%$ \\
\hline Ripetizione 4a & $.0 \%$ & $3.1 \%$ & $.0 \%$ & $1.2 \%$ \\
\hline Altro & $10.2 \%$ & $6.2 \%$ & $3.6 \%$ & $6.5 \%$ \\
\hline indecisione 37 & $18.4 \%$ & $18.4 \%$ & $14.2 \%$ & $17 \%$ \\
\hline & $100 \%$ & $100 \%$ & $100 \%$ & $100 \%$ \\
\hline
\end{tabular}

È da notare che il 46.2\% di coloro che non sanno indicare la professione più adatta o ideale per loro (il $18 \%$ del campione), opta per una SMS. Inoltre il $25.5 \%$ dei ragazzi che scelgono le SMS non desiderano fare né una professione intellettuale o scientifica, né intermedia, né di impiegato amministrativo; questi casi si concentrano su coloro che: non sanno quale è la professione più adatta $\mathrm{a}$ loro o hanno un'idea ancora generica dell'ambito professionale o formativo adatto a loro. Questi dati indicano una scarsa riflessione e progettualità professionale per una buona fetta di coloro che scelgono una SMS, così come una scarsa conoscenza degli sbocchi professionali dei diversi percorsi formativi per coloro che frequentano una SMS ma vorrebbero intraprendere percorsi professionali per i quali esistono altre soluzioni formative.

\section{La percezione di possibili ostacoli}

Se dunque si rileva una importante propensione alla scelta di percorsi di maturità, non vi sono però preoccupazioni relative a possibili ostacoli che potrebbero intervenire su questo percorso (ottenimento della media necessaria, ottenimento di un posto di tirocinio, cambiamento nella motivazione, ...). Sono soprattutto i genitori di coloro che pensano di entrare in una SMS ad affermare, per l'86.4, che non vi saranno ostacoli o problemi per i loro figli; più realistici sono i giovani di questa categoria che per il $50 \%$ vedono possibili problemi. I genitori di coloro che scelgono un tirocinio in azienda non vedono possibili ostacoli o problemi per il 70\%, mentre i giovani sono meno ottimisti dei loro stessi genitori e vedono problemi per l' $80 \%$ dei casi, soprattutto legati, nel caso dell'apprendistato, al trovare un contratto di tirocinio per la professione scelta.

\section{Una "seconda scelta»?}

E stato domandato ad allievi e genitori quale percorso alternativo intraprenderebbero nel caso in cui la scelta segnalata portasse a degli insuccessi o non si presentasse conforme alle aspettative. Dalle risposte a questa domanda emerge chiaramente che il $70 \%$ dei giovani che hanno deciso di intraprendere un percorso di SMS ritenterebbe su questa strada, nonostante un possibile insuccesso/delusione. Per le altre opzioni rispettivamente solo il $30 \%$ (per le scuole professionali 
a tempo pieno) e il $40 \%$ per le scuole professionali con tirocinio in azienda ritenterebbero la medesima via. Le risposte dei genitori ricalcano queste principali differenze, sebbene con minore perseveranza per quanto concerne la ripetizione dell'anno in una SMS. (54\%). Questo dato esprime chiaramente come la scelta di una SMS risulta essere più persistente di quella professionale, anche a fronte di insuccessi.

\section{Le fonti di informazione}

Se fino a questo punto ci siamo concentrati sulla natura e sulle ragioni della scelta formativa; ci spostiamo ora sul reperimento delle informazioni necessarie a questa presa di decisione; in particolare ci si è domandati quali fonti di informazione siano state utilizzate dai giovani e quanto essi le percepiscano utili per effettuare una scelta formativa/professionale.

La percentuale maggiore di utilizzo (Cfr. Tabella 7) viene fatta registrare dagli opuscoli o brochure informativi (ad esempio la guida dell'UOSP «Scuola Media e poi?») (66.9\%). Quest'ultima percentuale, tuttavia, sorprende dal momento che questi materiali sono distribuiti e presentati in tutte le classi a tutti gli alunni secondo le pratiche rilevate presso gli orientatori e i docenti di classe. Seguono poi gli stages di orientamento (56.4\%), i siti internet specifici $(55.20 \%)$, gli eventi informativi (ad esempio Espo-Professioni) (55\%), le visite alle aziende $(33.10 \%)$ e la consultazione di offerte presenti in annunci ed inserzioni. In generale queste medie di utilizzo o partecipazione non rispecchiano l'universalità con cui tali misure vengono presentate ai giovani nei diversi contesti.

I giovani attribuiscono la maggior utilità tra le fonti/eventi di informazione agli stages di orientamento (5.81=Media) che risultano, nel 65\% dei casi, l'occasione pratica di una loro attivazione per la ricerca di informazioni. Parrebbe quindi che maggiore è la loro attivazione personale, la loro ricerca di informazione e maggiore parrebbe l'utilità che essi attribuiscono a quella esperienza. In generale i genitori percepiscono un'utilità maggiore di tutte queste fonti rispetto ai loro figli e si sentono mediamente molto ben informati a proposito di queste fonti (Media=6.83 su una scala da 1 a 9 ; DS $=1.8$ ).

Dal punto di vista dei figli, per il 97.1\% (Cfr. Tabella 8), il maggiore interlocutore con cui discutere della scelta sono i genitori, con i quali l'80\% dei giovani afferma di aver parlato della scelta «molte volte». 
Tabella 7. Percentuale di utilizzo delle fonti di informazione, utilità percepita e ricerca proattiva delle informazioni.

\begin{tabular}{|l|c|c|c|c|}
\hline Fonte & $\begin{array}{c}\text { Hai utilizzato } \\
\text { questa fonte? } \\
\text { “sı̀) }\end{array}$ & \multicolumn{2}{|c|}{$\begin{array}{c}\text { Utilità media percepita } \\
(1=\text { per niente utile; } \\
\text { 9=molto utile) }\end{array}$} & $\begin{array}{c}\text { Hai richiesto/cercato tu } \\
\text { queste informazioni? } \\
\text { (\% “sı̀) }\end{array}$ \\
\hline Brochure informative & $66.90 \%$ & allievi & genitori & allievi \\
\hline Stages di orientamento & $56.40 \%$ & 5.35 & 6.97 & $39.0 \%$ \\
\hline Siti internet & $55.20 \%$ & 5.33 & 7.94 & $64.4 \%$ \\
\hline Eventi informativi & $55.00 \%$ & 5.11 & 7.37 & $59.2 \%$ \\
\hline Visite alle aziende & $33.10 \%$ & 4.34 & 7.08 & $42.3 \%$ \\
\hline Annunci inserzioni & $22.70 \%$ & 3.44 & 5.38 & $40.8 \%$ \\
\hline
\end{tabular}

Tabella 8. Figure di riferimento e loro peso nella scelta.

\begin{tabular}{|l|c|c|}
\hline Persona di riferimento & Ne hai parlato con...? & $\begin{array}{c}\text { Quanto peso ha/hanno avuto } \\
\text { nella tua scelta? }\end{array}$ \\
\hline Genitori & $97.1 \%$ & $\begin{array}{c}\text { Punteggio medio totale su } \\
\text { una scala da 1 a 9 }\end{array}$ \\
\hline Orientatore di sede & $77.2 \%$ & 6.66 \\
\hline Compagni di scuola & $70 \%$ & 5.26 \\
\hline Docente di classe & $69.4 \%$ & 4.17 \\
\hline Parenti & $66.7 \%$ & 4.36 \\
\hline $\begin{array}{l}\text { Altre persone che mi hanno } \\
\text { raccontato la loro esperienza }\end{array}$ & $62 \%$ & 4.82 \\
\hline Amici fuori dall'ambito scolastico & $61.4 \%$ & 4.92 \\
\hline Altro docente & $51.2 \%$ & 4.17 \\
\hline Fratelli/sorelle & $40.9 \%$ & 3.7 \\
\hline Un orientatore esterno & $3.6 \%$ & 4.6 \\
\hline
\end{tabular}

I genitori risultano essere l'interlocutore che ha avuto maggior peso nella scelta dei giovani (6.66=Media; su una scala da 1 a 9; Cfr. Tabella 8), almeno dal punto di vista dei giovani stessi. Al contrario i genitori stessi non sentono di giocare un ruolo così centrale. Solo il 6\% dei genitori afferma di aver indirizzato i figli nella loro scelta. Oltre alla circolarità dell'informazione per cui, come evidenziato più sopra, i genitori risultano essere un'importante fonte di informazione per i figli e, seppur in misura minore, anche viceversa, emerge una scarsa coscienza dei genitori sul ruolo da loro assunto nel processo di scelta. Questa interpretazione trova conferma puntuale nelle interviste (vedi più oltre). 
Le ricadute e cambiamenti ad un semestre di distanza Volendo considerare il processo di transizione un processo complesso e cronologicamente dislocato su di un asse temporale, si è proceduto ad un secondo rilevamento sul medesimo campione degli allievi, volto a conoscere i) la realizzazione del progetto formativo espresso al termine del secondario I, ii) la tenuta e la costanza delle aspettative e rappresentazioni professionali, e iii) la soddisfazione percepita relativamente al corso di studi intrapreso. La Tabella 9 descrive la "coerenza» tra la scelta della fase 1 e la frequentazione effettiva delle scuole nella fase 2 .

Per quanto attiene la frequentazione effettiva, il 41.6 dei giovani rispondenti ad entrambe le fasi frequenta effettivamente una SMS, il 14.4\% una SPTP, e il 24.8\% una SPTA. Come mostrato nell'ultima riga della Tabella 9, tali dati si discostano dalla fase 1 , almeno, dal momento che i progetti formativi della prima fase erano così ripartiti: $42.9 \%$ per le SMS, $10 \%$ per le SPTP, e $22.4 \%$ per le SPTA. Si registra una diminuzione di frequentazione effettiva per le SMS, per le scuole professionali si registra un aumento (di 4 punti per le SPTP, e di 2 per le SPTA).

Il dato relativo alla coerenza di ciascun progetto formativo ci permette però di evidenziare degli "spostamenti» interessanti: la coerenza è espressa dalla percentuale determinata dall'incrocio fra lo stesso tipo di scuola. In primis è ravvisabile un certo scollamento tra le scelte delle fase 1 e la situazione nella fase 2 . Il $92.3 \%$ di coloro che prevedevano di frequentare una SMS in effetti la frequenta; il dato risulta più basso per le SPTP (84.6\%) e SPTA (78.6\%). Nel dettaglio vi è stato uno spostamento interessante per le SPTA, così riassumibile: le scuole professionali con tirocinio in azienda subiscono al loro interno uno spostamento del $14.3 \%$ verso altre soluzioni, uno spostamento del 3.6\% verso soluzioni temporanee, del 3.6\% verso le SPTP, per un totale di più del $20 \%$ che, partendo da questa opzione, si sposta verso altri percorsi. Al contempo ricevono la fetta più importante di quel pubblico che risultava indeciso tra due opzioni e di coloro che hanno cambiato idea rispetto al loro progetto iniziale (ricevendo il $29 \%$ della sua attuale popolazione da altre scelte).

Si sostanzia così l'ipotesi iniziale che vedeva nel professionale duale anche un bacino che riceve coloro che non avevano optato espressamente per questa scelta. Le bocciature potenziali sono state sottovalutate nella fase ex-ante, poiché solo un allievo aveva risposto di voler ripetere la $4^{\circ}$ media, mentre i ripetenti effettivi sono 9.

Per quanto concerne le percezioni dei giovani circa il percorso effettivamente frequentato, all'incirca 4 allievi su 5 hanno giudicato «Molto bene» e «Bene» il sentirsi in relazione con la scuola o la professione intrapresa. Le proporzioni di «Molto bene» variano significativamente per tutte le variabili considerate (bocciatura, sede scolastica, sesso, corsi attitudinali), ma in particolare coloro che 
hanno intrapreso un tirocinio in azienda si sentono per il 42.9\% "molto bene» rispetto al percorso scelto, mentre coloro che frequentano una SMS rispondono molto bene solo per il $17.3 \%$ dei casi, contro una media del $29 \%$ circa. Coloro che non hanno mai bocciato si collocano per il 77.5 tra le categorie «molto bene» e «bene»; mentre coloro che hanno alle spalle già una bocciatura si collocano per il $90 \%$ dei casi tra «bene» ed «indifferente».

Tabella 9. Coerenza tra progetti fase ex-ante e frequentazione effettiva fase ex-post.

\begin{tabular}{|l|r|r|r|r|r|r|r|}
\hline \multirow{2}{*}{$\begin{array}{l}\text { Fase ex-ante } \\
\text { Scelte }\end{array}$} & \multicolumn{7}{|c|}{ Frequentazione effettiva (fase ex-post) } \\
\cline { 2 - 8 } & SMS & SPTP & SPTA & $4^{\circ}$ media & Sol. temp & Altro & TOT. (N) \\
\hline SMS & $92.3 \%$ & $1.9 \%$ & $1.9 \%$ & $3.8 \%$ & $.0 \%$ & $.0 \%$ & 52 \\
\hline SPTP & $7.7 \%$ & $84.6 \%$ & $7.7 \%$ & $.0 \%$ & $.0 \%$ & $.0 \%$ & 13 \\
\hline Ripeterò $4 a$ & $.0 \%$ & $3.6 \%$ & $78.6 \%$ & $.0 \%$ & $3.6 \%$ & $14.3 \%$ & 28 \\
\hline Altro & $.0 \%$ & $.0 \%$ & $.0 \%$ & $100.0 \%$ & $.0 \%$ & $.0 \%$ & 1 \\
\hline SMS/SPTP & $11.1 \%$ & $.0 \%$ & $.0 \%$ & $22.2 \%$ & $.0 \%$ & $66.7 \%$ & 9 \\
\hline SMS/SPTA & $12.5 \%$ & $62.5 \%$ & $.0 \%$ & $25.0 \%$ & $.0 \%$ & $.0 \%$ & 8 \\
\hline SMS/Ripeterò $4 a$ & $50.0 \%$ & $.0 \%$ & $50.0 \%$ & $.0 \%$ & $.0 \%$ & $.0 \%$ & 2 \\
\hline SMS/Altro & $.0 \%$ & $.0 \%$ & $.0 \%$ & $100.0 \%$ & $.0 \%$ & $.0 \%$ & 1 \\
\hline SPTP/SPTA & $.0 \%$ & $.0 \%$ & $.0 \%$ & $.0 \%$ & $.0 \%$ & $100.0 \%$ & 2 \\
\hline SPTP/Altro & $.0 \%$ & $.0 \%$ & $80.0 \%$ & $.0 \%$ & $20.0 \%$ & $.0 \%$ & 5 \\
\hline SPTA/Altro & $.0 \%$ & $.0 \%$ & $50.0 \%$ & $50.0 \%$ & $.0 \%$ & $.0 \%$ & 2 \\
\hline SPTA/Ripeterò 4a & $.0 \%$ & $.0 \%$ & $100.0 \%$ & $.0 \%$ & $.0 \%$ & $.0 \%$ & 1 \\
\hline Numerosità & $.0 \%$ & $.0 \%$ & $.0 \%$ & $.0 \%$ & $100.0 \%$ & $.0 \%$ & 1 \\
\hline \% frequent.effettiva & $41.6 \%$ & $14.4 \%$ & $24.8 \%$ & $7.2 \%$ & $2.4 \%$ & $9.6 \%$ & 125 \\
\hline \% Scelte 1 ${ }^{\circ}$ Fase & $42.9 \%$ & $10.0 \%$ & $22.4 \%$ & $1.2 \%$ & & $23.70 \%$ & 170 \\
\hline
\end{tabular}

Si modifica anche la relazione intercorrente tra la rappresentazione professionale espressa nella prima indagine e quella della seconda fase: si riscontra un dimezzamento di coloro che dichiaravano di voler praticare una professione intellettuale o scientifica (da 30.2\% degli allievi a 15.4\%), che confluiscono nella categoria di chi non sa quale sia la professione più adatta a lui/lei (dal $7 \%$ al $13 \%)^{38}$.

\section{Analisi delle interviste}

A questi dati quantitativi principalmente relativi alle percezioni di genitori e allievi, occorre aggiungere una serie di considerazioni derivanti dalle interviste svolte con gli orientatori professionali e scolastici operanti nelle sedi, e con i coordinatori di sede per l'orientamento e che rappresentano un'interessante fonte di informazione per indagare l'organizzazione, i compiti e le attività svolte 
dai diversi attori istituzionali che ruotano attorno al processo di transizione. Tali professionisti sono infatti stati chiamati ad esprimersi - senza conoscere ancora i risultati dei questionari - i) sulle proprie pratiche professionali di informazione ed orientamento all'interno delle sedi e ii) e sulle ragioni che a loro modo di vedere sono alla base della problematicità della transizione I. Qui di seguito riportiamo una panoramica indicativa dei contenuti e del numero di citazioni per $\mathrm{i}$ temi trattati all'interno delle interviste semi-strutturate (in totale 6 interviste semi-strutturate della durata di un'ora l'una). Il corpus ottenuto dalla loro trascrizione è stato sottoposto ad un'analisi del contenuto con il supporto del software Atlas.ti. Si sono così individuate nel testo circa 280 porzioni significative di testo, a loro volta riconducibili a codici (ovvero categorie uniformi di contenuto), che presentiamo nella Tabella 10.

Tabella 10. Tematiche emergenti nelle interviste e numerosità delle relative citazioni.

\begin{tabular}{|l|l|r|}
\hline Categoria & \multicolumn{1}{|c|}{ Codice39 specifico } & $\begin{array}{c}\text { Numerosità } \\
\text { citazioni }\end{array}$ \\
\hline & & \\
\hline \multirow{2}{*}{$\begin{array}{l}\text { Descrizione delle pratiche } \\
\text { (tot. 125; 44.3\%) }\end{array}$} & Attività reali & 70 \\
\cline { 2 - 3 } & Ruoli nel settore orientamento & 43 \\
\cline { 2 - 3 } & Compiti & 12 \\
\hline \multirow{3}{*}{$\begin{array}{l}\text { Opinioni a proposito della } \\
\text { transizione }\end{array}$} & La transizione verso FP & 47 \\
\cline { 2 - 3 } (tot.157; 55.7\%) & La transizione verso SMS & 43 \\
\cline { 2 - 3 } & Il ruolo della famiglia & 30 \\
\cline { 2 - 3 } & Il ruolo del territorio & 18 \\
\cline { 2 - 3 } & I criteri della scelta & 19 \\
\hline Tot. & & 282 \\
\hline
\end{tabular}

1. Come si evince dalla Tabella 10, il $44.3 \% \%$ delle porzioni di testo rilevate è relativo alle pratiche professionali e ai ruoli professionali delle figure intervistate. A questo livello gli elementi principali emersi sono:

- il rilevamento di una mancanza di strategia condivisa e pianificata della collaborazione tra $i$ coordinatori di sede, gli orientatori professionali e $i$ docenti di classe con funzione di orientamento, così come delle competenze specifiche. Tale coordinamento è al momento lasciato all'iniziativa interna delle diverse figure e delle singole sedi. Una strategia di collaborazione e chiarificazione reciproca di ruoli, mansioni e competenze risulterebbe essenziale ai fini di una maggiore visibilità dei ruoli stessi nelle sedi scolastiche.

- La preoccupazione condivisa per la ricerca di nuove, più efficaci e maggiormente personalizzate strategie comunicative, a fronte delle forme più tradizionali legate ad una informazione generale, poco personalizzata (albo scolastico, comunicazioni a tutti gli allievi, a tutta la classe). Appare evidente la coerenza con i dati sull'utilità delle fonti rilevata tramite il questionario (Cfr. Tabella 7). 
2. Il restante $55.7 \%$ delle citazioni fa riferimento ad opinioni relative alle problematiche seguenti:

- per orientatori e coordinatori di sede, è esplicitata in maniera chiara la misconoscenza, da parte dei giovani, dei mestieri, dei possibili percorsi professionali e della relativa mobilità possibile all'interno del sistema professionale. Questo nodo critico porterebbe alla conseguente idealizzazione di alcune professioni (di più alto profilo), parallelamente allo screditamento di altre (di più basso profilo).

- Secondo gli intervistati, viene in generale attribuito scarso valore alla scelta di tipo professionale ${ }^{40}$. La scelta della formazione duale sembra essere per i giovani e le famiglie una scelta di ripiego, alla quale si approda, in alcuni casi, dopo svariati fallimenti in altri ordini di scuola ${ }^{41}$.

- Viene oggi accordata una preferenza evidente alle scuole medie superiori. Questo fenomeno sarebbe da porre in relazione con le elevate ambizioni ed aspettative dei giovani, non sempre commisurate alle loro reali competenze ${ }^{42}$. Inoltre, la scelta di una scuola media superiore non porrebbe il giovane di fronte alla scelta, ben più complessa, di una professione ${ }^{43}$.

- Il ruolo giocato dalla famiglia, secondo la prospettiva di questi operatori, è certamente centrale, ma non sufficientemente esplicitato. L'accompagnamento svolto dalla famiglia porta infatti implicitamente con sé una serie di valori quali la tradizione o la storia lavorativa di una famiglia, le rappresentazioni spesso obsolete e le elevate attese dei genitori che in diversi casi riconoscono nelle scuole medie superiori l'unica strada per la realizzazione personale 44 . Sembrano confermarsi le considerazioni fatte a proposito delle Tabelle 4 e 5 e del Grafico 1.

\section{Sintesi delle analisi e prospettive di intervento}

Le analisi quantitative e qualitative portano nel loro complesso a riconoscere l'emergere di quattro principali «aree critiche» che rappresentano, per la fase di sviluppo del progetto SCELTOplus, la base per l'ideazione e, in seguito per la sperimentazione di una serie di misure di intervento che vadano nella direzione di una maggiore consapevolezza professionale dei giovani nell'operare la loro scelta per il secondario II. Le considerazioni di sintesi della ricerca mettono in evidenza la necessità di intervenire a sostegno della transizione I grazie ad una meglio strutturata e più approfondita «educazione alla scelta» per i giovani e per le loro cerchie di influenza diretta (genitori) e indiretta (figure istituzionali che concorrono a facilitare il passaggio). Questo anche in considerazione del fatto che diverse delle ipotesi fondanti il progetto e la peculiarità della situazione ticinese per quanto riguarda la transizione I sono state sostanziate dalla ricerca. 
In modo particolare le «aree critiche» passibili di intervento possono essere riepilogate come segue:

1. l'area critica delle idealizzazioni ed attese;

2. l'area critica della progettualità e mobilità formativo-professionale;

3. l'area critica dei canali informativi;

4. l'area critica dei ruoli e delle strategie.

Le prime tre aree rispondono ad esigenze informative e contenutistiche, mentre l'area dei ruoli e delle strategie si colloca nell'ambito di una ottimizzazione dei processi organizzativo-istituzionali relativi all'orientamento.

1. L'area critica delle idealizzazioni e delle attese è sostanziata da alcuni dati emersi, ed in modo particolare da quelli relativi ai miti disfunzionali, alle credenze irrealistiche dei giovani (p. 297) e alle elevate aspettative di giovani e genitori relativamente alle funzioni assolte dalla professione nella vita degli individui (p. 296). Le lacune nella conoscenza di sé (p. 299) costituiscono in maniera ipotetica una base per la creazione di miti disfunzionali: una sovrastima od una sottostima delle proprie capacità ed attitudini può portare alla formulazioni di credenze non realistiche circa il proprio futuro professionale e/o formativo. L'assenza di conflitti tra genitori e figli (p. 300) sulle scelte relative al secondario II e sulle professioni ideali per i figli, mostra come il meccanismo delle attese e dei progetti ideali possa subire un processo di rinforzo positivo nella relazione tra genitori e figli, caratterizzata, appunto, da assenza di divergenze sul tema.

2. Per quanto concerne l'area della progettualità e della mobilità formativo-professionale, sono state misurate nella tassonomia del CDDQ delle difficoltà nella scelta relativamente a lacune nella conoscenza del processo di scelta e delle sue tappe (p. 300). I giovani sembrano non avere una piena conoscenza di quale sia il processo di una scelta di tipo professionale-formativo e delle fasi che questa implica. Emergono pure lacune nella conoscenza dei mestieri (peraltro sottolineate anche dagli orientatori e dai coordinatori di sede), dei possibili percorsi formativo-professionali, così come delle opportunità di mobilità presenti nel sistema formativo. Tale misconoscenza del mondo del lavoro è alla base delle idealizzazioni di alcune professioni (intellettuali e scientifiche) e della scarsa propensione verso altre. Vi sarebbe una polarizzazione ed una idealizzazione di alcuni tipi di professioni e la "fuga" da altre professioni (professioni dei servizi, della vendita, professioni intermedie, amministrative; Cfr. p. 295-296). Le rappresentazioni professionali di coloro che vorrebbero intraprendere professioni intellettuali o scientifiche è destinata però a mutare con più facilità rispetto a coloro che scelgono altri tipi di mestieri. Tali rappresentazioni, non confrontatesi con una reale scelta professionale, si presentano molto più labili, più ideali e meno realistiche (p. 305). 
La problematica della progettualità è risultata centrale anche per quanto concerne la mancanza di rappresentazioni professionali future per buona parte di coloro che scelgono una SMS (p. 300-301). Questo a sostenere l'ipotesi secondo la quale questa scelta rappresenta un ostacolo alla formazione di una vera scelta (o di progetti di scelta) di tipo più marcatamente professionale.

Da un punto di vista sistemico, inoltre, è bene evidenziare che $i$ percorsi di formazione professionale duale sono bacino di ricezione di coloro che non scelgono in prima istanza la via professionale, ma che vi approdano come ad una scelta di ripiego (p. 304). Il tirocinio in azienda rappresenta anche un bacino di popolazione «in uscita», dal momento che parte di coloro che si erano decisi per una formazione professionale duale, si trovano (per situazioni contingenti, mancanza di posti di tirocinio confacenti in primis) a non poter praticare tale scelta.

3. La terza area riguarda la problematica comunicativo-informativa.

Sono emersi in questo ambito alcuni limiti nell'utilizzo e nell'utilità percepita dei mezzi di informazione ai quali i giovani sembrano non fare riferimento in maniera diffusa, come invece parrebbe auspicato e praticato dalle figure istituzionali di riferimento (orientatori, coordinatori di sede, docenti di classe; (p. 302). Anche il ruolo giocato dalla famiglia pare in questo contesto di un certo rilievo: se per i giovani i genitori costituiscono il primo e più importante punto di riferimento nella maturazione della scelta, gli stessi genitori mostrano una scarsa consapevolezza riguardo al ruolo loro attribuito (p. 303).

Le analisi sintetiche di queste prime tre aree critiche hanno contribuito all'emergere di ipotesi di strategie d'intervento finalizzate a migliorare la «maturità vocazionale» (Denjean \& Julita, 2007) degli attori della transizione (dal pubblico target che sono gli allievi ai "pubblici veicoli» che sono i genitori, $\mathrm{i}$ docenti di classe, i coordinatori di sede e anche tutti i docenti) attraverso l'acquisizione di competenze sia relative alla struttura dei mestieri che alla consapevolezza di sé.

4. Per quanto attiene il livello organizzativo, la quarta area emersa dalla ricerca riguarda appunto i ruoli e le strategie degli attori istituzionali coinvolti.

Viene infatti rilevata la mancanza di una strategia condivisa e pianificata della collaborazione tra $i$ coordinatori di sede, gli orientatori professionali e $i$ docenti di classe con funzione di orientamento all'interno delle sedi scolastiche, così come delle competenze, dei ruoli e delle relazioni reciproche. Tale coordinamento è al momento lasciato all'iniziativa interna e singola di ciascuna sede (p. 306).

Su queste basi, che lasciano intravvedere buoni margini di intervento congiuntamente contenutistici e organizzativo-istituzionali, il progetto si prefigge nella sua fase di implementazione di elaborare, sperimentare ed implementare delle mi- 
sure di intervento e di ottimizzazione delle attività di orientamento, affinché le scelte dei giovani siano il più possibile meditate, consapevoli, mature e, per quanto possibile, libere da condizionamenti sociali e culturali.

\section{Note}

1 La sigla ISCED sta per International Standard Classification of Education. Su questa scala i livelli da 0 a 2 corrispondono all'incirca alla scuola dell'obbligo; il livello 3 rappresenta la scuola secondaria superiore, con le varianti a) della formazione accademica, b) della formazione professionale superiore e c) dell'entrata nel mondo del lavoro. Per una più ampia trattazione sulla classificazione ISCED dei livelli formativi, si veda http://www.uis.unesco.org/TEMPLATE/pdf/isced/ISCED_A.pdf.

2 Per una panoramica del sistema formativo svizzero si veda il sito della Conferenza dei Direttori Cantonali della Pubblica Educazione (CDPE) al link http://www.edk.ch/dyn/16237.php. Una specificazione delle possibilità formative transitorie la si trova nel rapporto «Etude approfondie sur les offres de formation transitoires entre scolarité obligatoire et formation professionnelle» (Egger, Dreher \& Partner, 2007). Gli autori sottolineano come sia sempre più in crescita la fascia di giovani che sono in difficoltà nel momento del passaggio dal livello secondario I al secondario II. Secondo questo studio, ogni anno, da 2000 a 2500 allievi non riescono ad entrare in una formazione di livello secondario II o nella vita attiva. Per questa ragione sono sempre più di decisiva importanza le cosiddette e già citate misure o percorsi transitori, con i diversi accenti e offerte cantonali.

3 http://www.nahtstelle-transition.ch/fr

4 http://www.tree-ch.ch

5 http://www.tree-ch.ch/html_de/docs/tree_d.pdf

6 Si ricorda che in Ticino il secondario I è strutturato come «scuola media unica», con un primo biennio di osservazione e un secondo biennio di orientamento. Nel secondo biennio le materie di matematica e tedesco vengono offerte in due livelli: il primo «di base» per esigenze elementari e il secondo «attitudinale» per esigenze avanzate.

7 Cfr. http://www.bfs.admin.ch/bfs/portal/fr/index/themen/15/01/key/blank/01.html.

8 Cfr. http://www.bfs.admin.ch/bfs/portal/fr/index/themen/15/04/ind4.Docment.116736.xls

9 Per ottenere ulteriori dati su questo punto e, più in generale, sullo stato della formazione professionale in Svizzera, si veda «Formazione Professionale, Fatti e Cifre» del Dipartimento Federale dell'Economia. Ufficio Federale della Formazione Professionale e della Tecnologia. Berna. 2008.

http://www.formazioneprofessionaleplus.ch/files/pdf/fakten_und_zahlen_08_i.pdf

10 Esso viene definito come l'indicatore che «mesure le pourcentage des élèves de 9ème année de l'année précédente qui ont commencé immédiatement une formation professionnelle initiale (formation professionnelle élémentaire comprise), une formation générale (formation aux métiers de l'enseignement, école de maturité gymnasiale, école de degré diplôme, école de culture générale) ou une solution intermédiaire (non certifiante, à savoir 10e année, autre école de culture générale ou préapprentissage)» (OFS, 2007a, p. 47).

$11 \mathrm{E}$ interessante osservare che i tassi di transizione diretta sono stati rapportati al tasso di disoccupazione per regione linguistica: da cui emerge la correlazione inversa tra l'evoluzione nel tempo delle transizioni immediate e il tasso di disoccupazione. Sebbene tutte le regioni siano esposte alle stesse dinamiche (quando aumenta la disoccupazione, le transizioni immediate nell'apprendistato diminuiscono), per il Ticino le transizioni immediate verso l'apprendistato sono state sistematicamente inferiori alle altre regioni. (OFS, 2007b).

Si veda anche 
http://www.bfs.admin.ch/bfs/portal/fr/index/themen/15/08/dos/blank/14/11.html.

12 Le diminuzioni nei due settori citati sono solo parzialmente compensate da un aumento dell' $1 \%$ nelle Scuole Medico Tecniche e dello $0.7 \%$ nella formazione empirica.

13 In Ticino, per poter accedere alle scuole di maturità liceale senza dover sostenere esami di ammissione, è necessario: «a) media delle note nelle materie obbligatorie (inclusa la materia scelta nell' opzione capacità espressive e tecniche) di almeno 4,65, con al massimo un'insufficienza; b) frequenza dei corsi attitudinali di matematica e tedesco; c) avere ottenuto almeno il 4,5 in italiano.» (Consiglio di Stato della Repubblica e Cantone Ticino, Regolamento Scuola Media, 1996, Art. 65). Per questa ragione è possibile affermare che la scelta per il secondario II è per molti versi già prefigurata strutturalmente in precedenza: le condizioni sopracitate fanno sì che solo una parte della popolazione scolastica abbia la possibilità di passare direttamente alle SMS; coloro che infatti non presentano le caratteristiche menzionate possono scegliere principalmente tra le diverse opzioni del settore professionale.

14 All'interno della formazione professionale vi sono importanti affluenze da parte di coloro che hanno tentato altre strade di formazione, senza riuscirvi. Donati analizza come sia in calo «soprattutto il passaggio diretto scuola media-apprendistato, mentre appaiono in crescita le entrate negli apprendistati dopo aver tentato altre vie formative» (Donati, 1999, p.63). In particolare egli analizza che nella formazione professionale vi è sempre negli anni un flusso migratorio positivo (nel 1993/94 del +28\% per le Scuole Professionali Artigianali e Industriali; e nel medesimo anno per le Scuole Professionali Commerciali, vi è un flusso di 60 unità che hanno cambiato percorso): «in queste scuole convergono molti giovani reduci da esperienze formative segnate quasi sempre da un insuccesso altrove» (ivi, p.64).

$15 \mathrm{Si}$ veda: http://www.ti.ch/DFE/USTAT/DATI_CANTONE/15_formazione/tabelle/ T_150204_01C.html.

16 Concorre a questa propensione culturale in Ticino anche la vicinanza con la tradizione e la cultura italiane, orientate, come noto, verso le scuole di cultura generale ed in particolare verso i licei.

17 «Da mit der Platzierung des Individuums in der gesellschaftlichen Ordnung verbunden ist, kommt dem sozialen Aspekt des Selbst grössere Bedeutung zu als dem persönlichen. In die Berufswahl gehen Urteile über die Arbeitswelt ein, die sich im Wesentlichen auf das Sozialprestige und die Geschlechtstyp der Berufe beziehen» (ivi, 2006, p.21).

18 In Ticino la figura del "coordinatore di sede» all'interno delle sedi scolastiche del settore medio, rappresenta una figura di interfaccia tra l'Ufficio dell'Orientamento Scolastico e Professionale e le sedi stesse per tutto quanto concerne la divulgazione dell'informazione.

19 In questo contributo si riporta la parte di ricerca di un più ampio progetto di ricercaazione, finanziato nel 2008 dall'Ufficio Federale della Formazione e della Tecnologia, denominato «SCELTOplus». Il gruppo di progetto è composto dalle seguenti istituzioni: Società degli Impiegati di Commercio (SIC Ticino), promotrice del progetto stesso; Istituto Universitario Federale per la Formazione Professionale (IUFFP); Ufficio Cantonale dell'Insegnamento Medio del Dipartimento dell'Educazione, della Cultura e dello Sport (DECS); la Divisione della Formazione Professionale del DECS (DFP); La Camera di Commercio dell'Industria e dell'Artigianato del Canton Ticino (Ccia-TI); Ufficio cantonale dell'Orientamento Scolastico e Professionale (UOSP); Conferenza per la Formazione Continua degli Adulti (CFC); Conferenza Cantonale dei Genitori (CCG). Lo IUFFP è responsabile, in collaborazione con la direzione di progetto, della conduzione della ricerca.

20 Come sottolinea Bergzog (2008), infatti, la problematica delle rappresentazioni professionali/formative e delle aspettative che i giovani si creano a questo proposito sono un dato fondamentale: «Viele Jugendliche verlassen nach der neunten oder häufiger zehnten Klasse die Sekundarstufe I ohne, teils mit diffusen, teils sogar 'falschen' Vorstellungen von dem, 
was sie in Ausbildung und Beruf erwartet» (p. 10).

21 Il disegno di campionamento ha previsto la selezione di tre sedi scolastiche. Queste ultime sono state scelte sulla base del dato storico della transizione degli ultimi dieci anni e in base alla loro collocazione geografica: la prima sede di scuola media considerata, situata in una zona di valle (discosta), è caratterizzata da una propensione, nell'ultimo decennio, per le transizioni verso il tirocinio duale ( $35 \%$ ca.); la seconda sede di scuola media è situata in un distretto cittadino ed è caratterizzata, secondo lo storico dell'ultimo decennio, da una marcata propensione verso le SMS; la terza sede di scuola media è situata in una zona di confine e presenta una elevata propensione per le SMS e per le Scuole Professionali a Tempo Pieno.

22 L'9.3\% dei genitori non ha risposto al questionario.

23 Per quanto concerne le informazioni anagrafiche del campione degli allievi (popolazione rispondente allievi $=172$ ), quest'ultimo si presenta equamente diviso in femmine e maschi $(49.4 \%=$ maschi; $50.6 \%=$ femmine $)$. La nazionalità degli allievi presenta una percentuale media del $19.2 \%$ di allievi di nazionalità straniera e una media del $23.3 \%$ di lingua madre non italiana. Il $14.5 \%$ degli allievi ha già ripetuto una classe nel corso del curriculum scolastico. Il $62.6 \%$ degli allievi frequenta 2 corsi attitudinali, il $18.1 \%$ un solo corso attitudinale e il $19.3 \% 2$ corsi di base. Dal confronto tra sedi si evince una differenza significativa per quel che riguarda la nazionalità $(\mathrm{p}=0.029)$ e la lingua materna $(\mathrm{p}=0.004)$. La sede geograficamente più discosta (sede di valle) conta una proporzione significativamente più alta di svizzeri rispetto alle altre due sedi, mentre la sede del distretto urbano conta una proporzione significativamente inferiore di allievi di lingua materna italiana. Esiste una correlazione significativa tra il fatto di essere stato bocciato e il tipo e numero di corsi attitudinali o di corsi di base frequentati $(\mathrm{p}=0.000)$. La proporzione di allievi che ha frequentato 2 corsi base e un solo corso attitudinale è significativamente più elevata tra i bocciati, rispettivamente la proporzione di allievi che hanno frequentato due corsi attitudinali è significativamente inferiore.

24 Le categorie professionali qui utilizzate sono quelle dell'ISCO-88, International Standard Classification of Occupations. Cfr. http://laborsta.ilo.org/applv8/data/isco88e.html.

25 Nella tabella sono riportate le percentuali di risposta dei genitori che hanno indicato precisamente una professione (64.4\%). Non sono incluse qui le risposte di coloro che «non sanno» e che hanno soltanto una idea generica della professione adatta al figlio.

26 Il 35.6\% dei genitori ha un'idea soltanto generica (area professionale) della professione più adatta al figlio, contro il $16.8 \%$ dei giovani.

27 Nella tabella sono riportate le percentuali di risposta dei giovani che hanno indicato precisamente una professione (83.2\%). Non sono qui riportate le risposte di coloro che «non sanno" o che hanno soltanto una idea generica del proprio futuro professionale.

28 Per quel che concerne i genitori e la loro professione esiste una correlazione tra la sede scolastica e il tipo di professione esercitata dal genitore $(\mathrm{p}=0.018)$. La sede di valle conta proporzionalmente meno professioni intellettuali e scientifiche e professioni intermedie, e più personale dei servizi e venditori e artigiani e operai.

29 Per i confronti tra proporzioni è stato utilizzato il test del CHI quadrato.

30 Non sussistono a questo proposito differenze regionali.

31 Mancanza di prontezza nell'operare la scelta.

32 Mancanza di informazioni.

33 Informazioni incoerenti.

34 Per quanto concerne l'interpretazione della scala gli autori indicano che «a difficulty category with a mean response of 6.34 and above is «salient», below 3.33 is «negligible», and in-between are «moderate»» da http://kivunim.huji.ac.il/cddq/FAQ.htm. Una procedura di interpretazione più esaustiva e complessa, basata sulla salienza relativa delle difficoltà, $\mathrm{e}$ 
validata da una serie di esperti in orientamento, è stata sviluppata in Amir, Gati, e Kleiman (2008).

35 È da notare che coloro che dicono di voler ripetere l'anno presentano la media di miti disfunzionali maggiore (6.00).

36 È da notare che tali livelli di difficoltà variano in maniera considerevole se si considera il gruppo di coloro che hanno già operato una scelta e coloro che invece non hanno alcuna idea: l'indecisione professionale del secondo gruppo è pari ad un punteggio medio di 5.34, quella del primo gruppo è pari a 3.02. Anche i miti disfunzionali $(\mathrm{Rd})$ diminuiscono laddove la scelta è già stata fatta: 4.84 rispetto al punteggio medio di 5.50 di coloro che «non sanno cosa fare» l'anno successivo.

$37 \mathrm{Ci}$ si riferisce a tutte le combinazioni di indecisione rilevate.

38 A questo occorre aggiungere che 20 ragazzi non hanno risposto alla domanda relativa alla professione ideale.

39 Con il termine "codice» si identifica una categoria di citazioni che è simile per contenuto: si tratta di una sorta di raggruppamento di quelle porzioni di testo, all'interno dell'intero corpus di dati, che fanno riferimento ad un medesimo tema, contenuto, problematica.

40 «Purtroppo oggi la scelta dell'apprendistato è una scelta per difetto» [coordinatore X]. «Secondo me il prestigio oggi dell'apprendistato non è molto alto, nel senso che quando lo si presenta ai genitori occorre sempre far vedere che l'apprendistato non è una scelta di secondo rango, ma può essere una via che permette lo stesso, se ho le capacità, di realizzare anche uno studio superiore» [coordinatore $\mathrm{Y}$ ].

41 «Di quelli che poi provano con le scuole medie superiori, l'interessante sarà vedere quanti tentano e tornano più tardi all'apprendistato, perché l'età del tirocinio si è spostata di molto. Prima o poi vengono respinti, e poi arrivano all'apprendistato proprio per causa di forza maggiore e questo è triste» [orientatore $Z$ ].

42 "Ce ne sono tanti per il liceo, alcuni avevano delle aspettative alte ma non avevano una visione corretta della propria realtà nel senso che puntavano in alto ma non si accorgevano che forse non avevano anche i mezzi, con dietro magari anche i genitori che spingevano, allora hanno fatto delle scelte che io, e magari anche la docente di sostegno, non condividevamo molto in quanto vedevamo le difficoltà dell'allievo e avremmo visto piuttosto l'apprendistato...". [coordinatore XR].

43 «in alcuni casi la scuola media superiore perché... questo permette, permette di non fare una scelta, di non confrontarsi, di avere determinate condizioni e continuare con una formazione di cultura generale. Il rischio è quello di non fare un percorso di orientamento... si va avanti naturalmente fino ad un certo periodo, poi quando ci sono problemi...» [orientatore T].

44 «Sicuramente le aspettative dei genitori, quello ha un grosso influsso, vediamo con la scuola media, tante volte sono i genitori che decidono "tu farai il liceo" perché vedono nel liceo l'unica strada, la strada migliore per un futuro vincente» [coordinatore Q]. 


\section{Bibliografia}

Albert, E. (2007). Wie beeinflusst das Bildungssystem die Übertrittsprozesse von der Schule in den Beruf? Soziologisches Institut der Universität Zürich.

Amir, T., Gati, I. \& Kleinman, T. (2008). Understanding and interpreting career decision-making difficulties. Journal of Career Assessment 16 (3), 281-309.

Amos, J., Böni, E., Donati, M., Hupka, S., Meyer, T. \& Stalder, B. (2003). Wege in die nachobligatorische Ausbildung. Die ersten zwei Jahre nach Austritt aus der obligatorischen Schule Zwischenergebnisse des Jugendlängsschnitts TREE. Neuchâtel : BFS.

Amos, J. (2007). Transitions école-emploi : apports de la recherche TREE. In M. Behrens (Ed.), La transition de l'école a la vie active ou le constat d'une problématique majeure (pp. 43-53). Neuchâtel : IRDP.

Bergzog, T. (2008). Beruffängt in der Schule an. Die Bedeutung von Schülerbetriebspraktika im Rahmen des Berufsorientierungprozess. Bielefeld: Bertelsmann.

Denjean, P. \& Julita, J.-M. (2007) . Effets du programme «Éducation des choix» sur la connaissance de soi. Lindécis. Repères pour l'approche trouver/créer de l'orientation tout au long de la vie: aux sources de l'éducation des choix 68, 66-86.

Donati, M. (1999). Volevi veramente diventare ciò che sei? La formazione dei giovani dopo la scuola media. Bellinzona: Ufficio studi e ricerche.

Donati, M. \& Lafranchi, G. (2007). Formazione sì. Lavoro anche? I percorsi formativi e professionali dei giovani: fra strategie individuali e logiche di sistema. Bellinzona : Ufficio studi e ricerche.

Egger, Dreher \& Partner AG. (2007). Étude approfondie sur les offres de formation transitoires entre scolarité obligatoire et formation professionnelle. Rapport. Bern: OFFT.

Gati, I., Krausz, M. \& Osipow, S. H. (1996). A taxonomy of difficulties in career decision making. Journal of Counseling Psychology 43, 510-526.

Ghisla, G. (2003). La scuola ticinese: un «Sonderfall» prezioso. In AAVV (Ed.), Il Ticino nella Svizzera (pp. 381-453). Locarno: Armando Dadò.

Ghisla, G., Bonoli, L. \& Loi, M. (Eds.). (2008). Economia della formazione professionale. Torino: UTET.

Guidotti, C. \& Rigoni, B. (2007a). Censimento degli allievi 2006/2007. Bellinzona: Ufficio studi e ricerche.

Guidotti, C. \& Rigoni, B. (2007b). La scuola ticinese in cifre. Edizione 2007. Bellinzona: Ufficio studi e ricerche.

Herzog, W., Neuenschwander, M. \& Wannack, E. (2006). Berufswahlprozess. Wie sich Jugendliche auf ihren Beruf vorbereiten. Bern: Haupt.

Masdonati, J. (2007). La transition entre école et monde du travail. Préparer les jeunes à l'entrée en formation professionnelle. Bern: Peter Lang.

Mühlemann, S., Schweri, J., Winkelmann, R. \& Wolter, S. (2007). An empirical analysis of the decision to train apprentices. LABOUR: Review of Labour Economics and Industrial Relations, 21 (3), 419-441.

Neuenschwander, M. P. et al. (2005). Schule und Familie - was sie zum Schulerfolg beitragen. Bern: Haupt.

OFS. (2007a). Mosä̈que de l'éducation en Suisse. Les indicateurs de la formation 2007. Neuchâtel: OFS.

OFS. (2007b). Perspectives de la formation. Scénarios 2007-2016 pour le degré secondaire II. Neuchâtel: DFI-OFS.

Pagnossin, E. \& Armi, F. (2008). Recherches suisses sur les transitions entre la formation et le monde du travail depuis les années 1980. Neuchâtel: IRDP.

UFFT. (2008). Formazione professionale, fatti e cifre. Berna: UFFT-DFE.

Wolter, S. \& Schweri, J. (2004). Ökonomische Aspekte der Organisation einer Berufslehre. Zeitschrift für Berufs- und Wirtschaftspädagogik, 100 (1), 13-25. 
Parole chiave: Transizione, Tirocinio in azienda, CDDQ, Orientamento scolastico e professionale

\section{Übergänge nach der obligatorischen Schule: wie sich Tessiner Jugendliche entscheiden}

\section{Zusammenfassung}

Der nationale Vergleich macht deutlich, dass Jugendliche im Kanton Tessin weniger häufig eine berufliche Ausbildung nach der obligatorischen Schule wählen. Der vorliegende Beitrag diskutiert eine Pilotstudie, die den Übergang von der Sekundarstufe I in die Sekundarstufe II im Kanton Tessin beobachtet. Insbesondere wird das Entscheidungsverhalten in Richtung duale Berufsbildung unter die Lupe genommen.

Auf der Basis des Career Decision Difficulties Questionnaire wurden Berufswünsche und -wahlen, Motive, Kriterien und Schwierigkeiten am Ende der vierten Oberstufe bei 170 Schülerinnen und Schülern erhoben und erfasst. Zusätzlich wurden die Eltern, die Berufsberater/innen, die Schulleiter/innen sowie die Lehrer/innen befragt. Die Daten der Erhebungszeitpunkte (Ende der Oberstufe, sechs Monate später) verweisen im schulischen Kontext auf etliche Interventionsmöglichkeiten im Berufswahlprozess der Jugendlichen: dies sowohl auf organisational-institutioneller wie auch auf inhaltlich-didaktischer Ebene.

Schlagworte: Übergänge, Berufsbildung, CDDQ, Berufswahlprozesse

\section{La transition après l'école obligatoire: les choix des jeunes au Tessin}

Résumé

Après l'école obligatoire, le pourcentage des jeunes tessinois choisissant un parcours de formation professionnelle en apprentissage est sensiblement inférieur à la moyenne nationale suisse. Dans la présente contribution, il s'agit de rendre compte d'une recherche visant à comprendre les modalités de transition entre secondaire I et secondaire II au Tessin, les problèmes soulevés en particulier en relation avec le choix de parcours de formation professionnelle en entreprise (apprentissage).

Par le biais du Career Decision Difficulties Questionnaire (CDDQ), les choix effectués, les difficultés, les raisons sous-jacentes et les critères en jeu ont été relevés auprès d'environ 170 élèves à la fin de l'école obligatoire, de leurs parents, des consultants de l'orientation professionnelle et scolaire et des professeurs de classe. Pour l'orientation professionnelle et scolaire, la recherche - qui a recueilli des données à la fin de la dernière année scolaire du secondaire I (juin 2008) et 
six mois après - a permis de définir quelques pistes d'interventions possibles concernant l'éducation au choix des jeunes. Ces interventions portent sur des aspects institutionnels et organisationnels et sur des contenus didactiques.

Mots clés: Transition, apprentissage en entreprise, Career Decision Difficulties Questionnaire CDDQ, orientation professionnelle et scolaire

\title{
Transitions into post-compulsory education: the choices of youth in the Ticino
}

\begin{abstract}
In comparison to other Swiss cantons, youth in the Ticino choose less frequently to join vocational education after compulsory education. This article presents a pilot study on the transition from lower to upper secondary education in the canton Ticino. Different forms of transition and problematic issues compared to those in vocational education are under scrutiny.

Based on the Career Decision Difficulties Questionnaire, data from 170 students have been collected on their vocational aspirations and choices, motives, criteria and difficulties at the end of the fourth year of lower secondary education. In addition, parents, career counsellors, head masters and teachers have been interrogated. The data from two surveys (end of lower secondary school, six month later) refer to potential interventions in the process of vocational choice in the education context: on the organisational-institutional level as well as on the content-methodological level.
\end{abstract}

Key words: Transition, vocational education, Career Decision Difficulties Questionnaire $(\mathrm{CDDQ})$, process of vocational choice 